Commentary/M azur $\&$ Booth: Testosterone and dominance

\title{
Honour subcultures and the reciprocal model
}

James Steele

Department of Archaeology, University of Southampton, Southampton SO17

1BJ, England. tjms@soton.ac.uk

Abstract: Tests of models of reciprocal interactions of testosterone and behaviour patterns in honour subcultures, if based on adult samples measured at a single point in time, would be aided by measures of behaviour in such samples that indirectly index basal testosterone levels at earlier developmental ages, for example, hand preference and other measures of cerebral dominance. Such modelsraisequestionsabout the social preconditions of honour subcultures, and their indirect effects on health.

M azur \& B ooth $(M \& B)$ make a convincing case for the involvement of testosterone $(T)$ in male dominance-seeking behaviour and for the reciprocal nature of the interaction. $M$ ore detail on the possible mechanisms involving $\mathrm{T}$ in the mobilization of physiological and cognitive resources would be useful. (I sn't it significant that increased T can rapidly elevate rates of cellular metabolism in muscle tissue [Tsai \& Sapolsky 1996]?) One recurrent psychological finding is an effect of elevated T on visual-spatial abilities ( $\mathrm{H}$ ampson 1995; Janowsky et al. 1994), but its precise nature is controversial. In this respect, the literature on T and dominance seeking in adults has much in common with the literature on fetal $\mathrm{T}$ and subsequent adult hand preference. $L$ efthandedness may be influenced by fetal T levels (Geschwind \& Galaburda 1987) and may also be associated with enhanced spatial cognition in adult males (Annett 1992); however, findings from longitudinal studies are inconsistent and hard to interpret in simple terms (see, e.g., Grimshaw et al. 1995).

An indirect test of the hypothesis linking fetal $\mathrm{T}$ levels (and thus, by implication, postnatal basal T) with subsequent handedness involves assessment of interactions between hand preference and immune status in children or adults. This follows from the wellknown association between elevated $\mathrm{T}$ and suppression of immune function in vertebrates generally (Zuk 1996). T is implicated in the development of male secondary sexual characteristics, such as the red comb of the cockerel, and it has been proposed that, despite their immunosuppressive effects, high T levels are maintained in males as a result of female sexual selection for male ornamentation. Other things being equal, we would therefore expect prolonged elevated T levels to be associated with poorer health status even in humans, although the relationship is not a simple one, because some individuals will have compensatingly high baseline levels of immunocompetence.

It follows from this that we would also expect to find, in "honour subcultures," a suppressive effect of chronically elevated T levels on immune status and thus on health, although, among individuals within the population, great variability may still exist in the strength of any such effect. However, unless the chronically elevated T levels characterised as the product of an "honour subculture" among young adult males in the reciprocal model somehow influence the fetal hormonal environment of their offspring, we would not expect to see atypical patterns of adult male hand preference or other measures of cerebral dominance. If hand preference or any other measures of cerebral dominance were among the measures recorded in either of the large military samples discussed by $M \& B$, then perhaps this further, indirect test of the reciprocal model could be carried out using adult data from a single point in time. [See commentary of Coren, this issue.]

Would $M \& B$ elaborate on the social preconditions of honour subcultures? The sample of 4,462 army veterans among whom M azur (1995) found an apparent effect on T and on behavioural deviance of participation in an inner-city, low-educational-attainment honour subculture is the same sample of 4,462 army veterans among whom D abbs (1992), B ooth and D abbs (1993), and Booth and Osgood (1993) found correlations between elevated T and low occupational status, marital dissatisfaction, and criminality. These correlations could therefore be due to latent socio- 
Response/Booth $\&$ M azur: Testosterone and dominance

economic variables (mediated by the interaction of age, race, and educational level, in the terms of the veterans' survey), rather than to the effects of $T$ on behaviour. 